\title{
A Novel Pre-Screening Kit for Cerebral Amyloid Deposition Using QPLEXTM-Alz Plus Assay Kit: Application to Cognitively Normal Individuals
}

\section{Dongjoon Lee}

Seoul National University College of Medicine Jong-Chan Park

Seoul National University College of Medicine

Keum Sim Jung

QuantaMatrix Inc., Seoul

Jiyeong Kim

QuantaMatrix Inc., Seoul

Ji Sung Jang

QuantaMatrix Inc., Seoul

\section{Sunghoon Kwon}

QuantaMatrix Inc., Seoul

\section{Min Soo Byun}

Seoul National University Bundang Hospital

\section{Dahyun Yi}

Seoul National University Hospital

\section{Gihwan Byeon}

Seoul National University Hospital

Gijung Jung

Seoul National University Hospital

\section{Yu Kyeong Kim}

SNU SMG Boramae Medical Center: Seoul National University Seoul Metropolitan Government Boramae Medical Center

\section{Dong Young Lee}

Seoul National University Hospital

\section{Sun-Ho Han}

Seoul National University College of Medicine

Inhee Mook-Jung ( $\square$ inhee@snu.ac.kr)

Seoul National University College of Medicine https://orcid.org/0000-0001-7085-4085 
Keywords: Alzheimer's disease, cognitively normal individual, Pittsburgh compound B, cerebral amyloid deposition, blood-based biomarker, QPLEXTM Alz plus assay kit

Posted Date: March 11th, 2021

DOI: https://doi.org/10.21203/rs.3.rs-277508/v1

License: (1) This work is licensed under a Creative Commons Attribution 4.0 International License. Read Full License 


\section{Abstract}

\section{Background}

The deposition of beta-amyloid $(A b)$ in the brain precedes the onset of symptoms such as cognitive abnormality in Alzheimer's disease (AD); therefore, it is best to detect Ab accumulation early as possible.

\section{Methods}

We previously reported that $Q P L E X^{T M}$ Alz plus assay could be used for pre-screening of Ab accumulation in the brain. In this study, we applied QPLEX ${ }^{T M}$ Alz plus assay kit to the cognitively normal (CN) group for the brain Ab positivity. Total $221 \mathrm{CN}$ participants with or without brain Ab were included.

Results

Receiver operating characteristics (ROC) curve analysis showed that the discrimination power reached 0.878 area under curve (AUC) with $69.7 \%$ sensitivity and $98.4 \%$ specificity in the CN group over 65 years.

Conclusions

We suggest that QPLEX ${ }^{T M}$ Alz plus assay is a useful method for pre-screening brain $A b$ in the CN group, especially over 65 years, to prevent disease progression by early detection of disease initiation.

\section{Background}

Alzheimer's disease (AD) is the most prevalent form of the disease that causes dementia, afflicting more than 40 million individuals worldwide (1). There are many causes of Alzheimer's disease (2-4), but undoubtedly the primary pathology of $A D$ is severe amyloid deposition in the brain (5). Multiple methods in assessing brain amyloid levels have been developed over the past years, including the use of Pittsburgh B compound positron emission tomography (PiB-PET) (6). However, due to limited accessibility of PiB-PET, ongoing trials have been made to find surrogates for PiB-PET, such as blood biomarkers that reliably indicate the brain amyloid level (7). Without the need for burdensome cerebrospinal fluid (CSF) extraction or high-cost brain imaging, numerous advantages of blood-based diagnosis are drawing the attention of researchers, clinicians, and the elderly population.

Several studies have shown changes in blood biomarker levels during the onset and progression of Alzheimer's disease $(8,9)$. Blood biomarkers show changes in level long before cognitive impairment, which means that overall systemic changes occur before brain malfunction (10). Moreover, the fact that brain amyloid deposition precedes cognitive abnormalities emphasizes the need for screening to be performed in cognitively normal (CN) individuals (8). Asymptomatic cerebral amyloidosis becoming a new criterion for preclinical AD, PiB-PET positive individuals, regardless of their cognitive state, are at risk of developing the symptoms (11). In this context, the prediction of $A D$ progression based on blood 
biomarkers could be beneficial to those $\mathrm{CN}$ individuals prone to progress to mild cognitive impairment $(\mathrm{MCl})$ stage by providing preventive measures and aiding in early intervention.

In our previous studies, we discovered a novel blood-based biomarker panel to predict cerebral amyloid deposition $(12,13)$ and developed a pre-screening platform for PiB-PET positivity using this panel $(14)$. Here we show that QPLEX ${ }^{T M}$ Alz plus assay kit can be utilized to distinguish $C N$ individuals with or without amyloid burden. The results show that the kit can be used to pre-screen amyloid deposition in the brain when no apparent cognitive abnormalities are present to identify individuals susceptible to $A D$ progression due to the amyloid burden in the brain.

\section{Methods}

\section{Participants}

In total, 221 participants were included in this study. All participants were cognitively normal. These individuals were recruited as part of the Korean Brain Aging Study for the Early diagnosis and prediction of Alzheimer's disease (KBASE). All participants were given appropriate clinical and neuropsychological assessments according to the KBASE assessment protocol. Participant recruitment, clinical diagnosis criteria, and further information are detailed in our previous report (15).

\section{Ethical approval}

All participants and (where applicable) their legal representatives read and confirmed the informed consent documents. This study was approved by the Seoul National University Hospital Institutional Review Board (IRB).

\section{PIB-PET}

All participants underwent PiB-PET scans using a 3.0T PET-MR scanner (Siemens Healthineers, Erlangen, Germany). Each participant was intravenously injected with $555 \mathrm{MBq}$ of [11C] PiB (450-610MBq) PET tracer, which enabled visualization of cerebral amyloid deposition. The automatic anatomic algorithm determined the degree of amyloid accumulation, which was calculated by the standardized uptake value ratio (SUVR). Four regions of interest (ROIs) in the brain were the lateral temporal, lateral parietal, posterior cingulate-precuneus, and frontal regions. If the SUVR value was 1.4 or higher for at least one of the four ROIs, the individual was defined as PiB-positive ( $\mathrm{PiB}+$ ). Additional information on the imaging protocols is provided in our previous paper (15).

\section{Blood sampling}

All fasting blood samples were collected at 9:00 AM. Whole-blood samples were gathered in K2 EDTA tubes (BD Vacutainer Systems, Plymouth, UK) and centrifuged at $700 \mathrm{~g}$ for 5 minutes at room temperature (RT). Then the supernatant was collected, the centrifugation was repeated, and the tubes were stored at $-80^{\circ} \mathrm{C}$. 
All quantification method is detailed in our previous paper (14). Briefly, the QPLEX $X^{T M}$ kit utilized Quantamatrix's multiplex diagnostics platform (QMAP; Quantamatrix Inc., Seoul, Republic of Korea) (16). First, human plasma samples were diluted in the diluent buffer and incubated with the coded beads and biotin-conjugated detection antibodies. The immunocomplexes were washed twice with washing buffer on a Biotek-510 magnetic wash station (Biotek, VT, USA). Diluted R-phycoerythrin-conjugated streptavidin was added to each well. After three washes, the immunocomplexes were resuspended in $100 \mu \mathrm{l}$ of washing buffer by tapping and were analyzed automatically by the QMAPTM system.

\section{Monotone regression spline analysis}

Analyses for monotone penalized regression splines were performed to identify the relationship between each biomarker response and the imaging biomarkers (17). Monotone curves were generated by the smoothing spline method with the number of four knots. To effectively show comparisons between different QPLEX ${ }^{T M}$ biomarkers, their levels have been transformed to z-scores. Participant's age acted as a proxy for progression time.

\section{Statistical analyses}

GraphPad Prism 8 (San Diego, CA, USA) and Medcalc 17.2 software (Ostend, Belgium) were used. Comparison analyses between two variables were conducted by independent $t$-test or analysis of covariance (ANCOVA) with correction for age and sex. Pearson's correlation analysis method was used for correlation analyses. Logistic regression, followed by receiver operating characteristic (ROC) curve analysis was performed to calculate the discriminatory power, sensitivity, and specificity for the biomarker panels. The formulas, coefficients, and constants could be optimized since there were appropriate outliers and various logistic regression models. By using the values of variance inflation factors (VIF), multicollinearities were checked. All statistical outliers were excluded from the cohort according to Grubb's double-side outlier test $(p<0.05)$.

\section{Results}

\section{Categorization of participants}

The cognitively normal (CN) participants were classified as CN- (PiB-PET negative, $n=185)$ and $C N+$ (PiBPET positive, $n=36$ ) groups. The details were shown in Table 1. There were no significant differences in sex, education level, mini-mental state examination (MMSE) score, apolipoprotein E $₫ 4$ (ApoE4) positivity, and clinical dementia rating (CDR) scores. In addition, the demographic data according to their agegroups (young, mid-age, and old-age) were shown in Supplementary Table 1. 
We had previously reported that our QPLEX ${ }^{T M}$ biomarkers (beta-amyloid1-40, Ab1-40; periostin, POSTN; galectin-3 binding protein, LGALS3BP; angiotensin-converting enzyme, ACE) showed significant differences between age-matched (age $>55$ years old) $\mathrm{PiB}^{-}$and $\mathrm{PiB}^{+}$participants (14). In the current study, our purpose was to utilize the biomarkers to distinguish whether amyloid accumulates within the $\mathrm{CN}$ group ( $\mathrm{CN}$ - vs $\mathrm{CN}+$; cognitively normal participants of all ages). Before the start, we tested the effects of age and sex, which are generally known as confounders of $A D$, on our biomarkers (Figure 1A and 1B). Note that we only used $\mathrm{CN}$ - samples for Figure 1 to exclude the intervention of $A D$ pathology from testing the confounders (age, sex). Interestingly, our monotone regression spline analysis found three different curve patterns of biomarkers according to aging (Figure 1A). The cut-off dividing lines (42 years old, 65 years old) separating age-groups were determined by the vertexes of each biomarker curve (POSTN and ACE showed the first vertex at the point of 42 years old; POSTN, ACE, and LGALS3BP showed the second vertex at the point of 65 years old; Ab1-40 showed unclear vertex). According to this cut-off criterion, we defined three age-groups as Young, Mid-age, and Old-age groups. Interestingly, the second cut-off point (65 years old) is exactly matched with the standard age for late-onset AD (Figure 1A) (18). Next, we identified that there were no differences in the biomarkers between male and female (Figure 1B). Furthermore, all of the biomarkers showed significant correlations between them (Figure 1C) but with low variance inflation factors ( VIF < 2), which means they are in the most suitable situation to be used as variables for the logistic regression.

Age-dependent approaches for identifying the correlation between the QPLEX ${ }^{T M}$ markers and cerebral amyloid deposition

Since the QPLEX ${ }^{T M}$ biomarkers showed different patterns between the age groups, we checked relationships between the biomarkers and cerebral amyloid deposition (standard uptake value ratio; SUVR) for each age-group (Figure 2). The correlations between cerebral amyloid deposition and the QPLEX ${ }^{T M}$ biomarkers were not significant in Young and Mid-age groups. In the Old-age group, we confirmed that periostin (POSTN) and galectin-3 binding protein (LGALS3BP) levels in blood showed remarkably significant correlations with cerebral amyloid deposition (Figure 2C). Thus, we decided to focus on the Old-age group and further investigated whether the QPLEX ${ }^{T M}$ biomarkers can be utilized to discriminate PiB-PET positivity within this group. Young and Mid-age groups were not appropriate for this analysis because they had a too small number of PiB+ participants (Supplementary Table 1). However, since this model was only limited to old-age participants, we thought it was an insufficient model for satisfying our goal, which targets all ages of $\mathrm{CN}$ participants. Therefore, we decided to generate new models for all ages with the correction for covariates (age, sex) to compensate for the age-dependent difference in the level of biomarker pattern and the significant difference of age between $\mathrm{CN}$ - and $\mathrm{CN}+$ (Supplementary Figure 1-2).

Relationship between the QPLEXTM biomarkers and cerebral amyloid deposition in Old-age group (>65 years) 
First, we performed a comparison analysis between $\mathrm{CN}$ - and $\mathrm{CN}+$ in the Old-age group. Although the biomarkers did not show any differences between the sexes (Figure 1B), we included this variable for a covariate because it has also been a well-known confounder of AD diagnosis (19). Interestingly, two biomarkers, periostin (POSTN) and galectin-3 binding protein (LGALS3BP) showed significant differences between $\mathrm{CN}$ - and $\mathrm{CN}+$ (Figure 3A-D, left). The overall level of POSTN in blood was significantly increased in individuals with cerebral amyloid deposition above the threshold, whereas LGALS3BP showed the opposite tendency. Partial correlation analysis with correction for age and sex also revealed a significant association between the blood biomarker levels and cerebral amyloid deposition (Figure 3A-D, right). POSTN was positively correlated with cerebral amyloid deposition (Pearson's correlation coefficient $\mathrm{R}=$ 0.2186 , P-value $=0.0333$ ), whereas LGALS3BP was negatively correlated (Pearson's correlation coefficient $\mathrm{R}=-0.2626$, P-value $=0.0094)$. When all age-groups were combined, all $Q P L E X^{T M}$ biomarkers except for ACE showed significant differences and correlations, shown in Supplementary Figure 1. Furthermore, the results from the analysis of covariance (ANCOVA) showed that All $\mathrm{CN}^{+}$group had significantly different levels of Ab1-40, POSTN, and LGALS3BP than All CN' group (Supplementary Table 2). Our results showed the possibility of the QPLEX ${ }^{T M}$ biomarkers as variables for the cerebral amyloid deposition, except for ACE.

Discriminative ability of the QPLEX ${ }^{T M}$ Alz plus assay for $C N-v s C N+$ in Old-age group (>65 years)

Before further analyses for the practical use, we had to determine whether Ab1-40 and ACE could be used as variables because they did not show the significant results from the previous analyses (Figure 2-3, Supplementary Table 3). However, when we performed multiple regression analysis on all CN groups, we found that all QPLEX ${ }^{T M}$ biomarkers, including Ab1-40 and ACE, showed a significant correlation with cerebral amyloid deposition (SUVR) in every combination of variables (Table 2). Thus, we decided to include Ab1-40 and ACE in our biomarker panel because they were verified to be useful variables in explaining cerebral amyloid deposition level, based on multiple regression analysis. Subsequently, we performed logistic regression and receiver operating characteristic (ROC) curve analysis to identify the discriminative ability of our QPLEX ${ }^{T M}$ Alz plus assay (Figure 4A). When three ROC curves were compared with each other, we found adding QPLEX ${ }^{T M}$ biomarkers dramatically increased area under curve (AUC) ( 0.622 to 0.878 , Curve I vs Curve III; 0.684 to 0.878 , Curve II vs Curve III) (Figure 4B). Each graph had high sensitivities or specificity (Curve I, $82.4 \%$ sensitivity and $42.9 \%$ specificity; Curve II, $52.9 \%$ sensitivity and $73.5 \%$ specificity; Curve III, $69.7 \%$ sensitivity and $98.4 \%$ specificity) (Figure 4 C). When all age-groups were combined, QPLEX ${ }^{T M}$ Alz plus assay still retained its discriminative ability, which is shown in

Supplementary Figure 2. Therefore, the results suggest that $Q P L E X^{T M}$ Alz plus assay can also be used for pre-screening amyloid deposition in the brain even when there are no apparent symptoms of cognitive disorders.

\section{Discussion}


The lack of disease-modifying treatment is the major problem for $A D$, and the most efficient solution for this disease would be the early diagnosis in the initial stage of pathogenesis. Preclinical AD is a widely accepted disease stage that shows the initiation of brain pathology and silent symptoms (11). Advanced technology enables identifying preclinical $A D$, such as functional imaging of $P E T, M R I$, and cerebrospinal fluid (CSF) $A \beta$, tau, and p-tau (20). However, these modern technology diagnoses have difficult access due to high cost and invasive procedures $(21,22)$. Easily accessible biomarkers for diagnosis are desperate in the stage of preclinical $A D$ for early detection and prevention of disease progression.

In this study, cognitively normal PiB-PET negative individuals of a broad age range were included. We performed a monotone regression spline analysis from these samples in which participants' age acted as a proxy for time (Figure 1A). This enabled us to observe the general trend of changes in QPLEX ${ }^{T M}$ blood biomarkers with aging in the absence of AD pathology. With participants ensured to be normal under a specific set of rules such as PiB-PET and clinical dementia rating, we could indirectly observe how our biomarkers undergo overall systemic changes under normal physiological conditions. Interestingly, the monotone regression spline curve's vertexes segmented the population into three age-groups with a distinct trend of $Q P L E X^{T M}$ biomarkers. Further correlation analyses focusing on individual age-groups were performed, which revealed a significant correlation in the Old-age group (Figure 2). Based on these results, along with the fact that 65 -years is a clinical standard in sporadic $A D$ and individuals in this age group are most vulnerable to be afflicted by dementia, we primarily focused on the Old-age group but also performed the same analyses as a whole (including all age-groups) in parallel.

Comparison analyses revealed that blood levels of POSTN and LGALS3BP were significantly different according to PiB-PET status in Old-age and Ab1-40 was added to the list when analyzed as a whole (Figure 3, Supplementary Figure 1). These biomarkers also showed a correlation with the level of amyloid deposition in the brain, which supports these blood markers' appropriateness as indicators of brain status. Multiple regression analyses in which the QPLEX ${ }^{\mathrm{TM}}$ biomarkers serve as significant variables in the models further support this notion (Table 2, Supplementary Table 3). ROC curve analyses show the biomarkers' discriminative ability and conclude that QPLEX ${ }^{T M}$ Alz plus assay is apt to be used for prescreening purposes (Figure 4, Supplementary Figure 2).

Recently, we discovered the diagnostic blood biomarkers for $\operatorname{AD}(13,14)$ and reported the diagnostic accuracy from a clinical trial with AD patients and age control group using QPLEX ${ }^{T M}$ Alz plus assay kit, which had been developed based on these biomarkers (14). In that study, we focused on the whole participants, including AD patients in old age and age-matched non-demented control groups (average age of $\mathrm{CN}-67.32 \pm 0.8, \mathrm{CN}+74.47 \pm 1.0$ ). Therefore, we were uncertain of the age at which individuals could apply the QPLEX ${ }^{T M}$ Alz plus assay kit for early detection of AD. Based on the fact that the pathological process of the amyloid deposition begins many years prior to clinical symptoms (20), it is best to test cerebral amyloid deposition as early as possible, especially in the case of individuals with a family history or other risk factors. Early detection of cerebral amyloid deposition with QPLEX ${ }^{T M}$ Alz plus assay kit during this preclinical or pre-symptomatic stage would be the best way to intervene to prevent or 
delay the clinical syndrome if possible. In this study, we tested whether this QPLEX ${ }^{T M}$ Alz plus assay kit is suitable for non-demented individuals not only in old-age but also as a whole, including the young and mid-age population, to detect cerebral amyloid deposition. Thus, we included 47 young (average age $32.04 \pm 0.9$ ), 77 mid-aged (average age $56.25 \pm 0.7$ ) and 97 old-aged (average age $74.06 \pm 0.5$ ) nondemented individuals (Supplementary Table 1). We proved that the QPLEX ${ }^{T M}$ Alz plus assay kit showed similar or even better efficiency of pre-screening in these groups (AUC 0.932), compared to the agematched, non-demented population reported in the previous publication (AUC 0.891) (14). Even though including age as a variable in ROC curve analysis showed a relatively high AUC value of 0.852

(Supplementary Figure 2) because many young PiB-PET negative individuals were included, significantly increased AUC value up to 0.932 was achieved in combination with QPLEX ${ }^{T M}$ Alz plus assay. This suggests the possibility of early screening for cerebral amyloid accumulation in a non-demented population using the kit.

\section{Limitations}

Age is a prominent factor in neurodegenerative diseases such as $A D$, and consequently, it was challenging to recruit cognitively normal participants of young age with brain amyloid burden. Thus, we could not perform ROC curve analyses on the Young and Mid-age group individually to dismiss the age effect, although we tried to adjust the factor by various measures. In the future, we plan to apply the kit to a larger cohort, and if the cohort has enough $\mathrm{CN}+$ individuals of young age, the applicability of our kit could be demonstrated more clearly.

\section{Conclusions}

This study demonstrates the ability of blood-based QPLEX ${ }^{T M}$ Alz plus assay in detecting PiB-PET positivity with high sensitivity and specificity. It supports using the kit for pre-screening cerebral amyloid deposition in cognitively normal individuals, especially in the old age-group with individuals aged over 65 years.

\section{Abbreviations}

AD: Alzheimer's disease; PiB-PET: Pittsburgh B compound positron emission tomography; CSF: cerebral spinal fluid; $\mathrm{CN}$ : cognitively normal; $\mathrm{MCl}$ : mild cognitive impairment; KBASE: Korean Brain Aging Study for the Early diagnosis and prediction of Alzheimer's disease; SUVR: standardized uptake value ratio; ROI: regions of interest; QMAP: Quantamatrix's multiplex diagnostics platform; ANCOVA: analysis of covariance; ROC: receiver operating characteristics; VIF: variance inflation factors; MMSE: mini-mental state examination; ApoE: Apolipoprotein E; CDR: clinical dementia rating; Ab1-40: beta-amyloid1-40; POSTN: periostin; LGALS3BP: galectin-3 binding protein; ACE: angiotensin-converting enzyme; AUC: area under curve

\section{Declarations}




\section{Ethical approval and consent to participate}

This project was approved by the Institutional Review Board (IRB) of the Seoul National University Hospital, South Korea, and the subjects of their legal representatives gave their written informed consent.

\section{Consent for publication}

Not applicable.

\section{Availability of data and materials}

Not applicable

\section{Competing interests}

The authors declare that they have no competing interests.

\section{Funding}

This work was supported by grants from the National Research Foundation (NRF) of Korea (NRF2019R1I1A1A01063525 to S-H.Han), from the Korea Health Technology R\&D Project through Korea Health Industry Development Institute (KHIDI), funded by the Ministry of Health \& Welfare, Republic of Korea (HI19C1132, HU20C0187, HU20C0198 to I. Mook-Jung), from the NRF (2018R1A5A2025964 to I. Mook-Jung, 2014M3C7A1046042 to D.Y.Lee), and from KHIDI (HI18C0630 \& HI19C0149 to D.Y.Lee).

\section{Author contributions}

D.L, J-C.P, and I.M-J conceptualized this study. D.L, J-C.P, K.S.J, J.K, J.S.J, and S.K performed experiments. M.S.B, D.Y, G.B, and G.J obtained all blood samples from the subjects and gathered all data. After PET scan data were obtained and collected, they were analyzed by Y.K.K, M.S.B, and D.Y. J-C.P analyzed statistics. D.L and J-C.P analyzed statistics and organized all figures and tables. D.Y.L provided the resources. D.L, J-C.P, and S-H.H wrote the original draft. S-H.H, D.Y.L, and I.M-J reviewed and edited the manuscript. All authors carefully read and approved the final manuscript.

\section{Acknowledgements}

We sincerely thank the patients, staffs of hospitals, and all volunteers for this project. We also thank EunHye Kim for the technical support for purifying and obtaining the plasma samples.

\section{References}

1. Collaborators GBDD. Global, regional, and national burden of Alzheimer's disease and other dementias, 1990-2016: a systematic analysis for the Global Burden of Disease Study 2016. Lancet Neurol. 2019;18(1):88-106.

2. Baik SH, Kang S, Lee W, Choi H, Chung S, Kim JI, et al. A Breakdown in Metabolic Reprogramming Causes Microglia Dysfunction in Alzheimer's Disease. Cell Metab. 2019;30(3):493-507 e6.

3. Choi H, Kim HJ, Yang J, Chae S, Lee W, Chung S, et al. Acetylation changes tau interactome to degrade tau in Alzheimer's disease animal and organoid models. Aging Cell. 2020;19(1):e13081. 
4. Cha MY, Cho HJ, Kim C, Jung YO, Kang MJ, Murray ME, et al. Mitochondrial ATP synthase activity is impaired by suppressed O-GlcNAcylation in Alzheimer's disease. Hum Mol Genet. 2015;24(22):6492504.

5. Murphy MP, LeVine H, 3rd. Alzheimer's disease and the amyloid-beta peptide. J Alzheimers Dis. 2010;19(1):311-23.

6. Uzuegbunam BC, Librizzi D, Hooshyar Yousefi B. PET Radiopharmaceuticals for Alzheimer's Disease and Parkinson's Disease Diagnosis, the Current and Future Landscape. Molecules. 2020;25(4).

7. Burnham SC, Faux NG, Wilson W, Laws SM, Ames D, Bedo J, et al. A blood-based predictor for neocortical Abeta burden in Alzheimer's disease: results from the AIBL study. Mol Psychiatry. 2014;19(4):519-26.

8. Jack CR, Jr., Knopman DS, Jagust WJ, Shaw LM, Aisen PS, Weiner MW, et al. Hypothetical model of dynamic biomarkers of the Alzheimer's pathological cascade. Lancet Neurol. 2010;9(1):119-28.

9. Hampel H, O'Bryant SE, Molinuevo JL, Zetterberg H, Masters CL, Lista S, et al. Blood-based biomarkers for Alzheimer disease: mapping the road to the clinic. Nat Rev Neurol. 2018;14(11):63952.

10. Zetterberg H, Burnham SC. Blood-based molecular biomarkers for Alzheimer's disease. Mol Brain. 2019;12(1):26.

11. Sperling RA, Aisen PS, Beckett LA, Bennett DA, Craft S, Fagan AM, et al. Toward defining the preclinical stages of Alzheimer's disease: recommendations from the National Institute on AgingAlzheimer's Association workgroups on diagnostic guidelines for Alzheimer's disease. Alzheimers Dement. 2011;7(3):280-92.

12. Park JC, Han SH, Lee H, Jeong H, Byun MS, Bae J, et al. Prognostic plasma protein panel for Abeta deposition in the brain in Alzheimer's disease. Prog Neurobiol. 2019;183:101690.

13. Park JC, Han SH, Cho HJ, Byun MS, Yi D, Choe YM, et al. Chemically treated plasma Abeta is a potential blood-based biomarker for screening cerebral amyloid deposition. Alzheimers Res Ther. 2017;9(1):20.

14. Park JC, Jung KS, Kim J, Jang JS, Kwon S, Byun MS, et al. Performance of the QPLEX Alz plus assay, a novel multiplex kit for screening cerebral amyloid deposition. Alzheimers Res Ther. 2021;13(1):12.

15. Byun MS, Yi D, Lee JH, Choe YM, Sohn BK, Lee JY, et al. Korean Brain Aging Study for the Early Diagnosis and Prediction of Alzheimer's Disease: Methodology and Baseline Sample Characteristics. Psychiatry Investig. 2017;14(6):851-63.

16. Chung SE, Park W, Park H, Yu K, Park N, Kwon S. Optofluidic maskless lithography system for realtime synthesis of photopolymerized microstructures in microfluidic channels. Appl Phys Lett. 2007;91(4).

17. Palmqvist S, Insel PS, Stomrud E, Janelidze S, Zetterberg H, Brix B, et al. Cerebrospinal fluid and plasma biomarker trajectories with increasing amyloid deposition in Alzheimer's disease. EMBO Mol Med. 2019;11(12):e11170.

18. Isik AT. Late onset Alzheimer's disease in older people. Clin Interv Aging. 2010;5:307-11. 
19. Podcasy JL, Epperson CN. Considering sex and gender in Alzheimer disease and other dementias. Dialogues Clin Neurosci. 2016;18(4):437-46.

20. Sperling RA, Karlawish J, Johnson KA. Preclinical Alzheimer disease-the challenges ahead. Nat Rev Neurol. 2013;9(1):54-8.

21. Wittenberg R, Knapp M, Karagiannidou M, Dickson J, Schott J. Economic impacts of introducing diagnostics for mild cognitive impairment Alzheimer's disease patients. Alzheimers Dement (N Y). 2019;5:382-7.

22. Zhang S, Smailagic N, Hyde C, Noel-Storr AH, Takwoingi Y, McShane R, et al. (11)C-PIB-PET for the early diagnosis of Alzheimer's disease dementia and other dementias in people with mild cognitive impairment (MCl). Cochrane Database Syst Rev. 2014(7):CD010386.

\section{Tables}

Table 1. Demographic data of the participants $(n=221)$

\begin{tabular}{|llll|}
\hline Characteristics $(n)$ & CN $^{-}(185)$ & CN $^{+}(36)$ & P-value \\
\hline Sex, M/F & $80 / 105$ & $20 / 16$ & $0.1755^{\dagger}$ \\
\hline Age, years, mean \pm SEM & $55.89 \pm 1.2$ & $74.47 \pm 1.0$ & $<0.0001^{*}$ \\
\hline Education, mean \pm SEM & $12.82 \pm 0.3$ & $12.08 \pm 0.8$ & 0.3846 \\
\hline MMSE raw score, mean \pm SEM & $27.78 \pm 0.2$ & $27.17 \pm 0.4$ & 0.1346 \\
\hline MMSE z score, mean \pm SEM & $0.48 \pm 0.1$ & $0.44 \pm 0.1$ & 0.7873 \\
\hline CDR (n) & $0(185)$ & $0(36)$ & - \\
\hline ApoE4 positivity, $\varepsilon 4+/ N(\%)$ & $40 / 185(22 \%)$ & $12 / 36(33 \%)$ & $0.1305^{\dagger}$ \\
\hline PiB (SUVR), mean \pm SEM & $1.09 \pm 0.004$ & $1.62 \pm 0.05$ & $<0.0001^{*}$ \\
\hline
\end{tabular}

Notes: ${ }^{*}$, significance by $t$-test; $\dagger$, significance by chi-squared test.

Abbreviations: $\mathrm{CN}$, cognitively normal; PiB, Pittsburgh compound B; - or +, PiB positivity; SEM, Standard Error of the Mean; n, number of participants; MMSE, Mini-Mental State Examination; MMSE z score, a revised value of the MMSE score with consideration for age, sex, and education level; CDR, Clinical Dementia Rating; ApoE, Apolipoprotein E; SUVR, standardized uptake value ratio; $\mathrm{N}$, total number of participants.

Table 2. Multiple regression analysis on cognitively normal individuals (including all age- groups) 


\section{QPLEX $^{\text {TM }}$ markers}

Dependent $Y \quad$ Cerebral amyloid deposition (SUVR)

Sample size

215

Coefficient of determination $\mathrm{R}^{2}$

0.1329

$\mathrm{R}^{2}$ - adjusted

0.1163

Multiple correlation coefficient

0.3645

Residual standard deviation

0.2210

$\begin{array}{lllllll}\text { Ind. variables Coefficient } & \text { Std. Error } & t & P & r_{\text {partial }} & r_{\text {semipartial }} & \text { VIF }\end{array}$

(Constant) 1.1778

\begin{tabular}{llllllll} 
Aß1-40 & 0.0009446 & 0.0003278 & 2.881 & 0.0044 & 0.1950 & 0.1851 & 1.131 \\
\hline LGALS3BP & -0.00002973 & 0.000008228 & -3.613 & 0.0004 & -0.2419 & 0.2322 & 1.211 \\
\hline ACE & -0.0004304 & 0.0001824 & -2.359 & 0.0192 & -0.1607 & 0.1516 & 1.465 \\
\hline POSTN & 0.006219 & 0.001873 & 3.320 & 0.0011 & 0.2233 & 0.2134 & 1.587
\end{tabular}

QPLEX ${ }^{\mathrm{TM}}$ markers + SeX + Age

Dependent $Y$ Cerebral amyloid deposition (SUVR)

Sample size

Coefficient of determination $\mathrm{R}^{2}$

$\mathrm{R}^{2}$ - adjusted

Multiple correlation coefficient

Residual standard deviation

Ind. variables Coefficient

(Constant) $\quad 0.9098$

$\begin{array}{llllllll}\text { AB1-40 } & 0.0006936 & 0.0003076 & 2.255 & 0.0252 & 0.1545 & 0.1344 & 1.158 \\ \text { LGALS3BP } & -0.00002887 & 0.000007703 & -3.747 & 0.0002 & -0.2515 & 0.2233 & 1.234 \\ \text { ACE } & -0.0003556 & 0.0001698 & -2.094 & 0.0375 & -0.1437 & 0.1248 & 1.476 \\ \text { POSTN } & 0.006376 & 0.001738 & 3.669 & 0.0003 & 0.2465 & 0.2186 & 1.588 \\ \text { Sex } & -0.02188 & 0.02842 & -0.770 & 0.4421 & -0.0533 & 0.0459 & 1.024 \\ \text { Age } & 0.005013 & 0.0008386 & 5.977 & <0.0001 & 0.3829 & 0.3562 & 1.026\end{array}$

215

0.2613

0.2400

0.5112

0.2049

$\begin{array}{llllll}\text { Std. Error } & t & P & r_{\text {partial }} & r_{\text {semipartial }} & \text { VIF }\end{array}$ 
QPLEX ${ }^{\mathrm{TM}}$ markers + SeX + Age + ApoE genotype

Dependent $Y$

Sample size

Coefficient of determination $\mathrm{R}^{2}$

$\mathbf{R}^{2}$-adjusted

Multiple correlation coefficient

Residual standard deviation

Ind. variables Coefficient

(Constant)

\begin{tabular}{llllllll|} 
AB1-40 & 0.0006864 & 0.0003043 & 2.256 & 0.0251 & 0.1549 & 0.1329 & 1.158 \\
\hline LGALS3BP & -0.00002854 & 0.000007619 & -3.746 & 0.0002 & -0.2519 & 0.2207 & 1.234 \\
\hline ACE & -0.0003705 & 0.0001680 & -2.205 & 0.0286 & -0.1515 & 0.1299 & 1.478 \\
\hline POSTN & 0.006314 & 0.001719 & 3.674 & 0.0003 & 0.2474 & 0.2165 & 1.589 \\
\hline Sex & -0.01911 & 0.02813 & -0.679 & 0.4977 & -0.0472 & 0.0400 & 1.025 \\
\hline Age & 0.005049 & 0.0008295 & 6.087 & $<0.0001$ & 0.3896 & 0.3587 & 1.027 \\
\hline ApoE & 0.07754 & 0.03259 & 2.379 & 0.0183 & 0.1632 & 0.1402 & 1.006 \\
\hline
\end{tabular}

Abbreviations: SUVR, standardized uptake value ratio; Ind., independent; VIF, variance inflation factor; A 1 1-40, amyloid beta 1-40; LGALS3BP, galectin-3 binding protein; ACE, angiotensin converting enzyme; POSTN, periostin; ApoE, apolipoprotein E

\section{Figures}


A

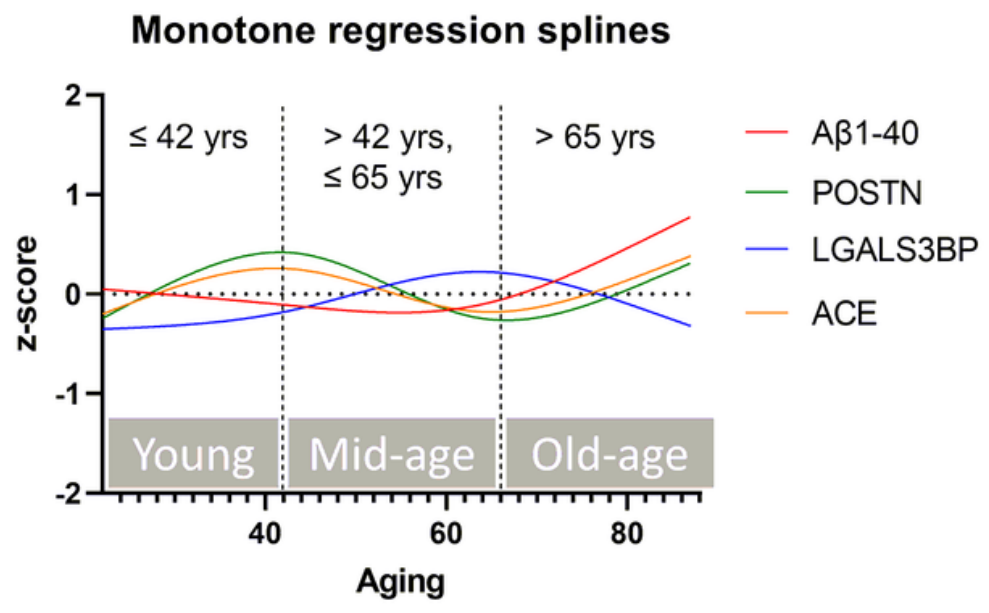

C

\begin{tabular}{|c|c|c|c|c|}
\hline & & R & R & \\
\hline & & 0.596 & 0.484 & \\
\hline $\begin{array}{l}\text { POSTN } \\
\text { ACE }\end{array}$ & 0.596 & .090 & 0.334 & 0.306 \\
\hline LGAL & 0.484 & 0.334 & & 0.276 \\
\hline A $\beta 1-40$ & 0.307 & 0.306 & 0.276 & \\
\hline & POSTN & ACE & LGAL & $A \beta 1-40$ \\
\hline POSTN & & $<0.0001$ & $<0.0001$ & $<0.0001$ \\
\hline ACE & $<0.0001$ & & $<0.0001$ & $<0.0001$ \\
\hline LGAL & $<0.0001$ & $<0.0001$ & & 0.0001 \\
\hline$A \beta 1-40$ & $<0.0001$ & $<0.0001$ & 0.0001 & \\
\hline
\end{tabular}

B
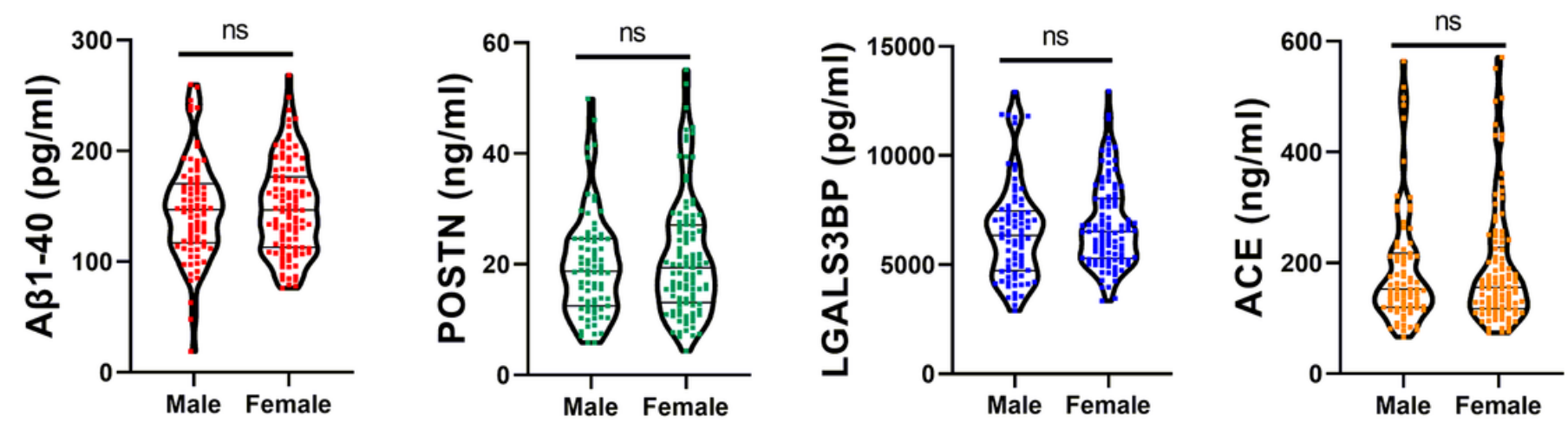

\section{Figure 1}

Characterization of QPLEXTM biomarkers in the cognitively normal participants without any intervention of $A D$ pathology Data from cognitively normal participants without brain amyloid (CN-) was used in this figure. (A) A monotone spline model for QPLEXTM biomarkers. The ages of participants acted as a proxy for time. The levels of QPLEXTM biomarkers have been transformed to z-score. The patterns of each biomarker show apparent differences according to age-groups (Young, Mid-age, and Old-age). Curves were created by a smoothing spline with the number of 4 knots. (B) No significant differences in QPLEXTM biomarkers between male and female. P-values were obtained from two-sided unpaired t-test. (C) Pearson's correlation between the QPLEXTM biomarkers. Colored and white boxes show R and Pvalues, respectively. We confirmed all variables had low variance inflation factors (VIF < 2). Abbreviations: Aß1-40, amyloid beta 1-40; POSTN, periostin; LGALS3BP(LGAL), galectin-3 binding protein; ACE, angiotensin converting enzyme 
A

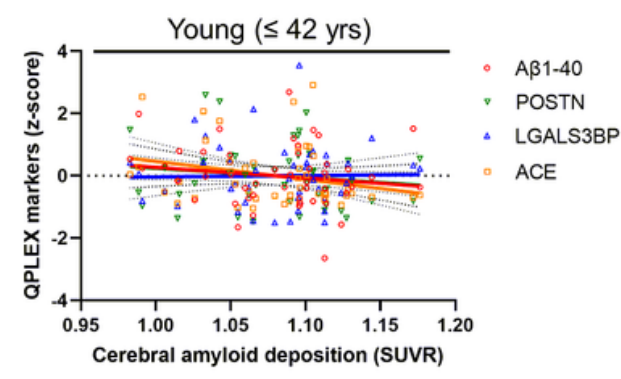

\begin{tabular}{lrrrr}
\hline & \multicolumn{1}{l}{ A 1 - 40 } & \multicolumn{1}{l}{ POSTN } & \multicolumn{1}{l}{ LGAL } & \multicolumn{1}{l}{ ACE } \\
\hline$R$ & -0.1538 & -0.1422 & 0.0195 & -0.2631 \\
$\mathrm{P}$ & 0.3019 & 0.3460 & 0.8965 & 0.0739 \\
\hline
\end{tabular}

B

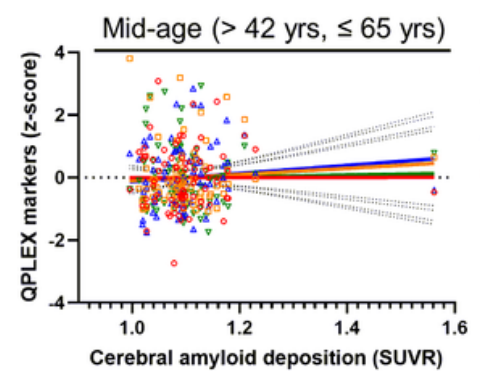

\begin{tabular}{rrrrr}
\hline & \multicolumn{1}{c}{ A $1-40$} & \multicolumn{1}{l}{ POSTN } & \multicolumn{1}{l}{ LGAL } & \multicolumn{1}{l}{ ACE } \\
\hline $\mathrm{R}$ & 0.0012 & 0.0196 & 0.0929 & 0.0716 \\
$\mathrm{P}$ & 0.9912 & 0.8658 & 0.4218 & 0.5415 \\
\hline
\end{tabular}

C

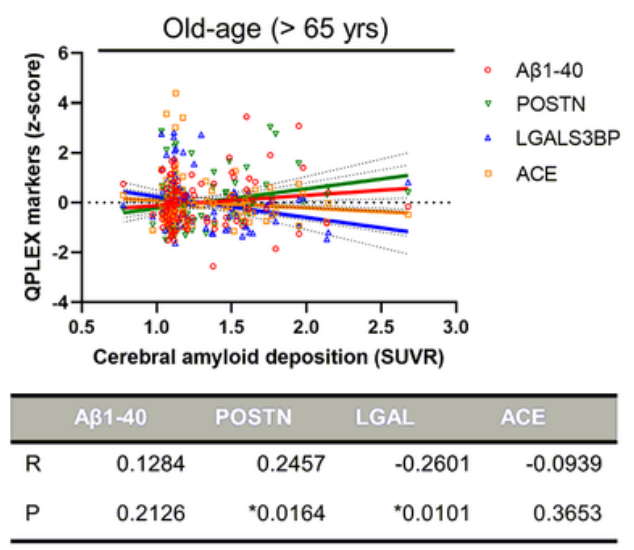

\section{Figure 2}

Age-dependent correlation between the QPLEXTM biomarkers and cerebral amyloid deposition Correlation between the QPLEXTM biomarkers and cerebral amyloid deposition (SUVR) according to the age-groups ((A) Young, (B) Mid-age, and (C) Old-age group). The levels of QPLEXTM biomarkers have been transformed to z-score. Only Old-age group showed noticeable significance. P-values were obtained from Pearson's correlation analysis (cut-off, ${ }^{*} p<0.05$, and ${ }^{* \star} p<0.01$ ). Abbreviations: SUVR, standardized uptake value ratio; A 1-40, amyloid beta 1-40; POSTN, periostin; LGALS3BP(LGAL), galectin-3 binding protein; $\mathrm{ACE}$, angiotensin converting enzyme 
A

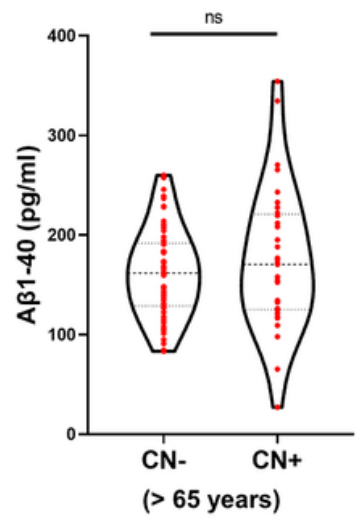

C

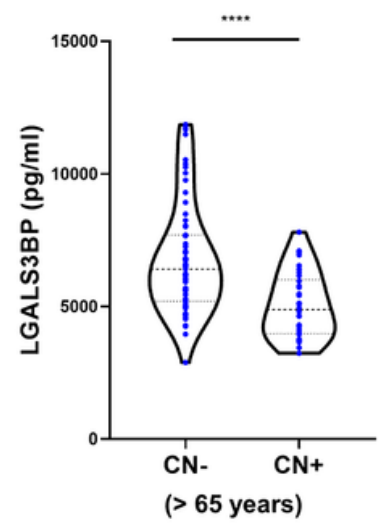

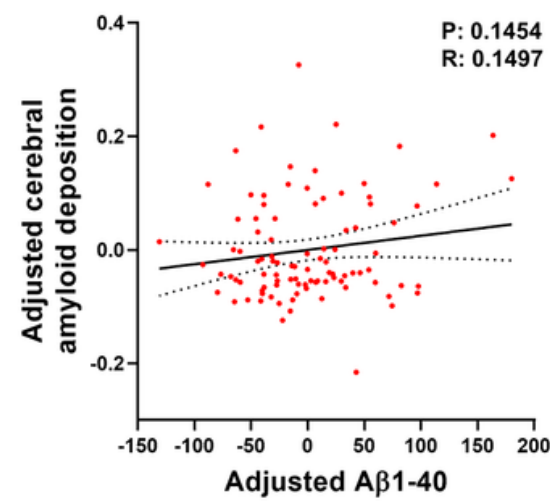

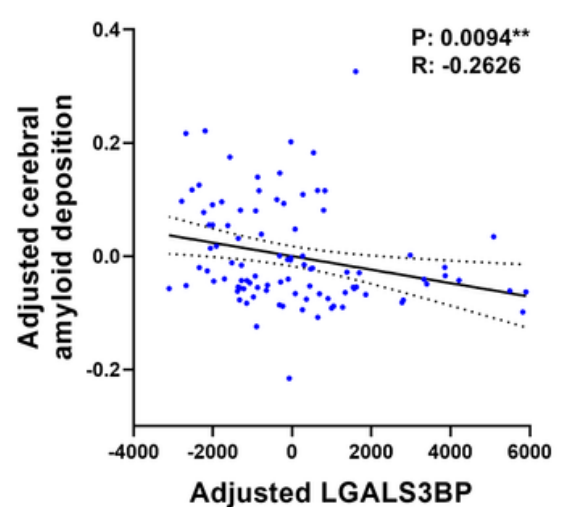

B
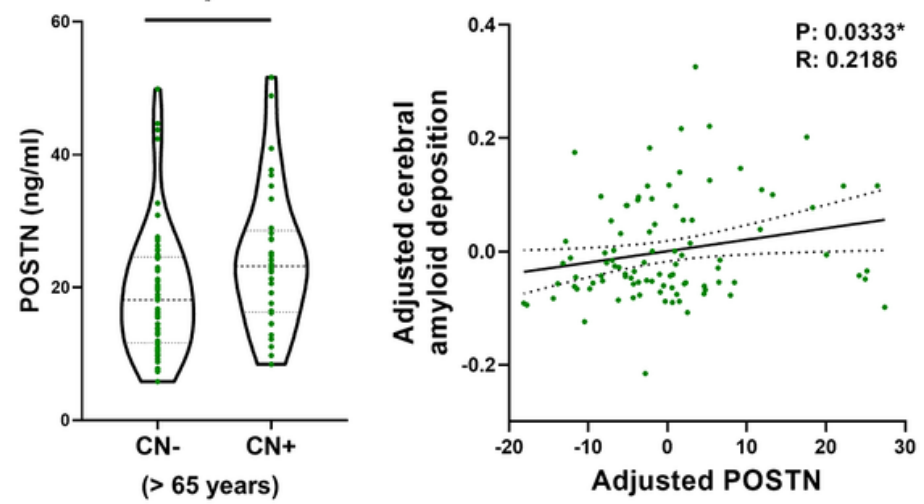

D
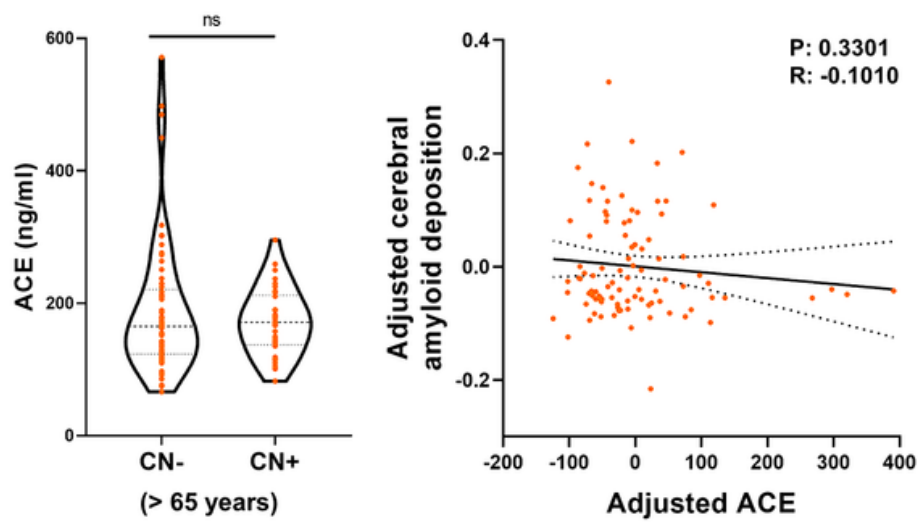

Figure 3

The QPLEXTM biomarkers and cerebral amyloid deposition for the Old-age group (>65 years) (A-D) Each left graph shows the comparison of QPLEXTM biomarker levels between $\mathrm{CN}$ - individuals and CN+ individuals within the Old-age group. Two-sided unpaired t-test was used. Each right graph shows partial correlation analysis with correction for age and sex. P-value cut-off, ${ }^{\star} p<0.05,{ }^{*} p<0.01,{ }^{\star \star \star} p<0.001$, and ${ }^{* \star *} p<0.0001$. P, Pearson's correlation P-value; R, Pearson's correlation coefficient. Dotted lines indicate $95 \%$ confidence intervals. Abbreviations: $\mathrm{CN}$, cognitively normal; - or +, PiB positivity; A 1 1-40, amyloid beta 1-40; POSTN, periostin; LGALS3BP, galectin-3 binding protein; ACE, angiotensin converting enzyme 
A

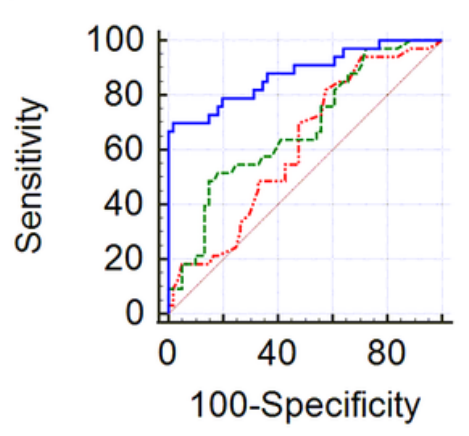

-.... Age + Sex (Curve I)

- Age + Sex + APOE + QPLEX (Curve III)
--- Age + Sex + APOE (Curve II)

B

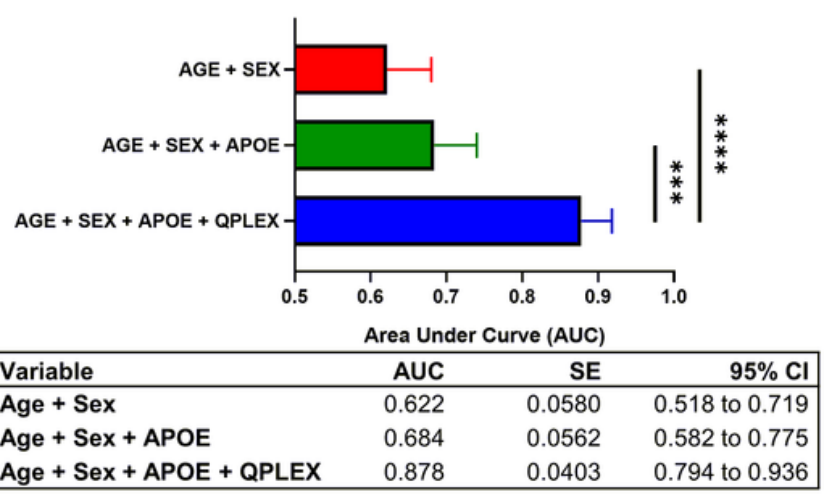

C
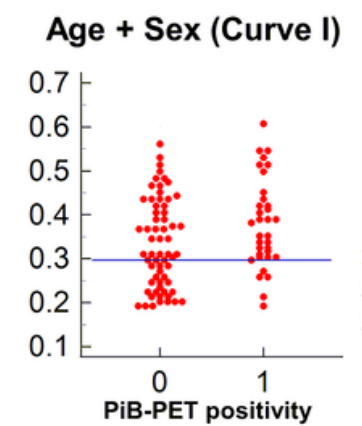

$>0.297$

Sens: 82.4

Spec: 42.9

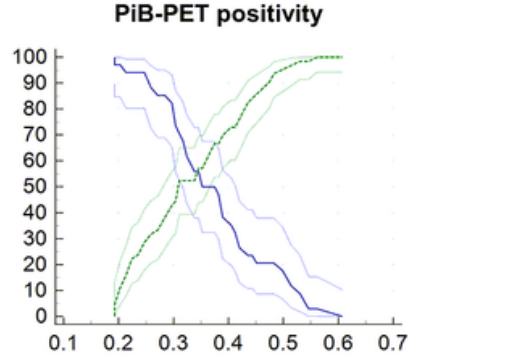

Age + Sex + APOE (Curve II)
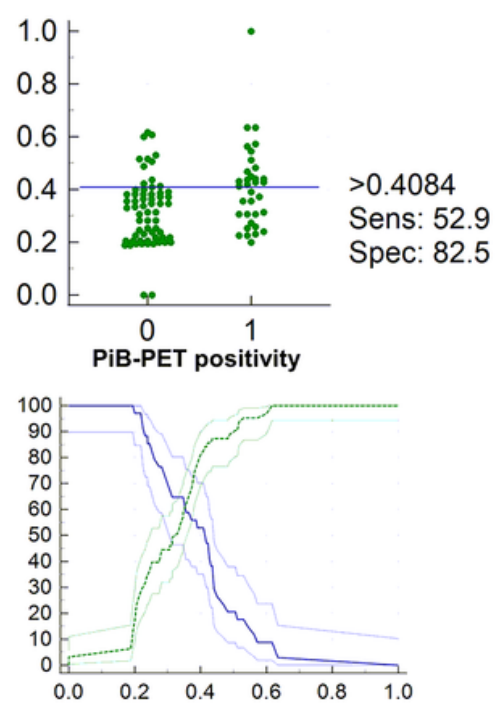

Age + Sex + APOE + QPLEX (Curve III)

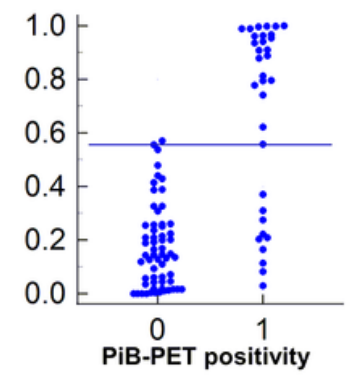

$>0.5566$

Sens: 69.7

Spec: 98.4

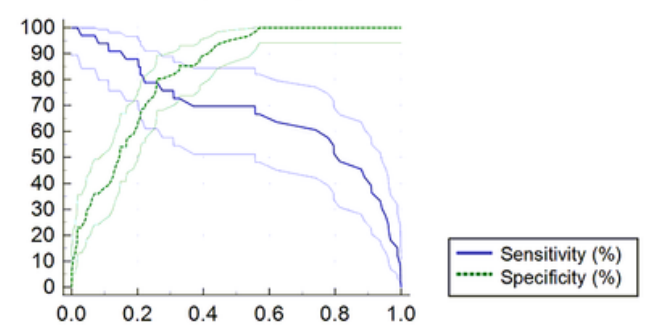

Figure 4

Receiver operating characteristic (ROC) curve analysis for PiB-PET positivity within Old-age group (>65 years) (A) ROC curve analysis for cognitively normal individuals grouped according to PiB-PET positivity. (B) Comparison of ROC curves with different combinations of age, sex, APOE genotype, and QPLEXTM biomarkers as variables. P-values were obtained from Delong's test $\left({ }^{*} p<0.05\right.$, ${ }^{\star *} p<0.01$, and ${ }^{\star \star *} p<$ 0.001). (C) Interactive dot diagram (upper) and graph of sensitivity, specificity, and their $95 \%$ confidence intervals plotted against the criterion values (lower). Abbreviations: APOE, Apolipoprotein E; AUC, area under curve; SE, standard error, $\mathrm{Cl}$, confidence interval

\section{Supplementary Files}

This is a list of supplementary files associated with this preprint. Click to download.

- 210224SUPPLEMENTARYINFORMATIONAZRT.docx 